(n)

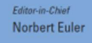
Journal of Nonlinear Mathematical Physics

\title{
Quantization of the dynamics of a particle on a double cone by preserving Noether symmetries
}

G. Gubbiotti, M.C. Nucci

To cite this article: G. Gubbiotti, M.C. Nucci (2017) Quantization of the dynamics of a particle on a double cone by preserving Noether symmetries, Journal of Nonlinear Mathematical Physics 24:3, 356-367, DOI:

https://doi.org/10.1080/14029251.2017.1341698

To link to this article: https://doi.org/10.1080/14029251.2017.1341698

Published online: 04 January 2021 


\title{
Quantization of the dynamics of a particle on a double cone by preserving Noether symmetries
}

\author{
G. Gubbiotti \\ Dipartimento di Matematica e Fisica, \\ Università degli Studi Roma Tre, \\ \& INFN Sezione di Roma Tre, 00146 Roma, Italy \\ gubbiotti@mat.uniroma3.it \\ M.C. Nucci \\ Dipartimento di Matematica e Informatica \\ Università degli Studi di Perugia \& INFN Sezione di Perugia \\ 06123 Perugia, Italy \\ nucci@unipg.it
}

Received 29 December 2016

Accepted 31 March 2017

\begin{abstract}
The classical quantization of the motion of a free particle and that of an harmonic oscillator on a double cone are achieved by a quantization scheme [M. C. Nucci, Theor. Math. Phys. 168 (2011) 994], that preserves the Noether point symmetries of the underlying Lagrangian in order to construct the Schrödinger equation. The result is different from that given in [K. Kowalski, J. Rembielński, Ann. Phys. 329 (2013) 146]. A comparison of the different outcomes is provided.
\end{abstract}

Keywords: Lie and Noether symmetries; motion of a particle on a double cone;classical quantization

PACS: 02.30.Hq, 02.20.Sv, 45.20.Jj, 03.65.Ge

\section{Introduction}

It has been known for more than sixty-five years that quantization and nonlinear canonical transformations have no guarantee of consistency [30]. For a more recent perspective see [2] where an up to date account of the various approaches to tackle canonical transformation is also provided.

In [17], [18], [19] a procedure which obviates the constraint imposed by the conflict between consistent quantization and the invariance of the Hamiltonian description under nonlinear canonical transformation was proposed. It is based on the preservation of Noether symmetries when going from classical to quantum mechanics. The quantization of classical problems is achieved by constructing a suitable time-dependent Schrödinger equation.

This method was reformulated in [8] for problems that are linearizable by Lie point symmetries, and also successfully applied to various classical problems: alternative Hamiltonian of an harmonic oscillator [17], second-order Riccati equation [18], dynamics of a charged particle in a uniform magnetic field and a non-isochronous Calogero's goldfish system [19], an equation related to a Calogero's goldfish equation [20], two nonlinear equations somewhat related to the Riemann problem [21], a Liénard I nonlinear oscillator [8], a family of Liénard II nonlinear oscillators [9], $N$ planar rotors and an isochronous Calogero's goldfish system [22]. 
If a system of second-order equations is considered, i.e.

$$
\ddot{\mathbf{x}}(t)=\mathbf{F}(t, \mathbf{x}, \dot{\mathbf{x}}), \quad \mathbf{x} \in \mathbb{R}^{N},
$$

that comes from a variational principle with a Lagrangian of first order, then the method that was first proposed in [17] can be summarized as follows:

(1) Find the Lie symmetries of the Lagrange equations

$$
\Upsilon=W(t, \mathbf{x}) \partial_{t}+\sum_{k=1}^{N} W_{k}(t, \mathbf{x}) \partial_{x_{k}}
$$

(2) Among them find the Noether symmetries

$$
\Gamma=V(t, \mathbf{x}) \partial_{t}+\sum_{k=1}^{N} V_{k}(t, \mathbf{x}) \partial_{x_{k}}
$$

This may require searching for the Lagrangian yielding the maximum possible number of Noether symmetries [23-26].

(3) Construct the Schrödinger equation ${ }^{\mathrm{a}}$ admitting these Noether symmetries as Lie symmetries, namely

$$
2 \mathrm{i} \Psi_{t}+\sum_{k, j=1}^{N} f_{k j}(\mathbf{x}) \Psi_{x_{j} x_{k}}+\sum_{k=1}^{N} h_{k}(\mathbf{x}) \Psi_{x_{k}}+f_{0}(\mathbf{x}) \Psi=0
$$

with Lie symmetries

$$
\Omega=V(t, \mathbf{x}) \partial_{t}+\sum_{k=1}^{N} V_{k}(t, \mathbf{x}) \partial_{x_{k}}+G(t, \mathbf{x}, \Psi) \partial_{\Psi}
$$

without adding any other symmetries apart from the two symmetries that are present in any linear homogeneous partial differential equation ${ }^{\mathrm{b}}$, namely

$$
\Psi \partial_{\Psi}, \quad \alpha(t, \mathbf{x}) \partial_{\Psi}
$$

where $\alpha=\alpha(t, \mathbf{x})$ is any solution of the Schrödinger equation (1.2).

If the system (1.1) is linearizable by a point transformation, and it possesses the maximal number of admissible Lie point symmetries, namely $N^{2}+4 N+3$, then in [6,7] it was proven that the maximaldimension Lie symmetry algebra of a system of $N$ equations of second order is isomorphic to $s l(N+$ $2, \mathbb{R})$, and that the corresponding Noether symmetries generate a $\left(N^{2}+3 N+6\right) / 2$-dimensional Lie algebra $g^{V}$ whose structure (Levi-Malćev decomposition and realization by means of a matrix

\footnotetext{
${ }^{\mathrm{a}}$ We assume $\hbar=1$ without loss of generality.

${ }^{b}$ In the following we will refer to those two symmetries as the homogeneity and linearity symmetries.
} 
algebra) was determined. It was also proven that the corresponding linear system is

$$
\mathbf{y}^{\prime \prime}(s)+2 A_{1}(s) \cdot \mathbf{y}^{\prime}(s)+A_{0}(s) \cdot \mathbf{y}(s)+\mathbf{b}(s)=0,
$$

with the condition

$$
A_{0}(s)=A_{1}^{\prime}(s)+A_{1}(s)^{2}+a(s) 1,
$$

where $A_{0}, A_{1}$ are $N \times N$ matrices, and $a$ is a scalar function.

Consequently if system $(1.1)$ admits $\operatorname{sl}(N+2, \mathbb{R})$ as Lie symmetry algebra then in [8] we reformulated the algorithm that yields the Schrödinger equation as follows:

Step 1. Find the linearizing transformation which does not change the time, as prescribed in nonrelativistic quantum mechanics.

Step 2. Derive the Lagrangian by applying the linearizing transformation to the standard Lagrangian of the corresponding linear system (1.3), namely the one that admits the maximum number of Noether symmetries ${ }^{\mathrm{c}}$.

Step 3. Apply the linearizing transformation to the Schrödinger equation of the corresponding classical linear problem. This yields the Schrödinger equation corresponding to system (1.1).

This quantization is consistent with the classical properties of the system, namely the Lie symmetries of the obtained Schrödinger equation correspond to the Noether symmetries admitted by the Lagrangian of system (1.1).

In [10] the problem of the quantization of the dynamics of a particle constrained on a double cone was considered.

A double cone of opening angle $2 \alpha$ with $\alpha \in(0, \pi / 2)$ is given by the cartesian equation:

$$
x^{2}+y^{2}-\cot ^{2}(\alpha) z^{2}=0 .
$$

A regular parametrization (except at the vertex $(0,0,0)$ ) for this surface is given by:

$$
\begin{aligned}
& x=r \sin (\alpha) \cos (\phi), \\
& y=r \sin (\alpha) \sin (\phi), \\
& z=r \cos (\alpha)
\end{aligned}
$$

with $r \in \mathbb{R}$ and $\phi \in[0,2 \pi)$.

Consequently a particle of mass $m$ constrained on a cone has the natural Lagrangian:

$$
L=\frac{1}{2} m\left(\dot{r}^{2}+\sin ^{2}(\alpha) r^{2} \dot{\phi}^{2}\right)-V(r, \phi),
$$

where $V(r, \phi)$ is the potential energy.

Without loss of generality we assume $m=1$. Also, since $\sin (\alpha)$ is a constant, we introduce another constant $k \in(0,1)$ such that $\sin (\alpha)=k$ in order to have neater expressions.

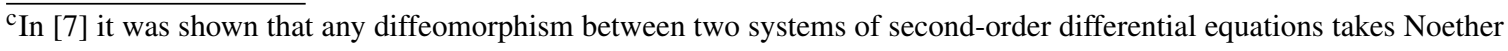
symmetries into Noether symmetries, and therefore the Lagrangian is unique up to a diffeomorphism. 
In [10] the authors addressed the quantization of two particular cases of the Lagrangian (1.7), namely the free particle, with Lagrangian ${ }^{\text {d }}$ :

$$
L_{\mathrm{f}}=\frac{1}{2}\left(\dot{r}^{2}+k^{2} r^{2} \dot{\phi}^{2}\right)
$$

and the radial harmonic oscillator, with Lagrangian ${ }^{\mathrm{e}}$ :

$$
L_{\mathrm{ho}}=\frac{1}{2}\left(\dot{r}^{2}+k^{2} r^{2} \dot{\phi}^{2}\right)-\frac{1}{2} \omega^{2} r^{2} .
$$

The radial harmonic oscillator was also considered in [3] as the classical motion of a particle on a cone under the influence of a central potential.

In this paper we apply the quantization algorithm that preserves the Noether symmetries to those two cases, and also determine the eigenvalues and the eigenfunctions of the obtained Schrödinger equations. We compare the results of our quantization method with those obtained in [10], and explain the differences that are due to symmetry breaking. This is the first instance of disagreement between the quantization method that preserves Noether symmetries and a quantization method, that looks for ad hoc self-adjoint operators, regardless of symmetry consideration.

\section{Quantization of a free particle on the cone}

The Lagrangian equations corresponding to the Lagrangian (1.8) are:

$$
\left\{\begin{array}{l}
\ddot{r}=k^{2} r \dot{\phi}^{2}, \\
\ddot{\phi}=-2 \frac{\dot{r} \dot{\phi}}{r} .
\end{array}\right.
$$

Using the REDUCE programs [16] we find that this system admits a fifteen-dimensional Lie point symmetry algebra, isomorphic to $\mathrm{sl}(4, \mathbb{R})$, generated by the following operators:

$$
\begin{aligned}
& \Gamma_{1}=\cos (k \phi) r\left(t \partial_{t}+r \partial_{r}\right) \\
& \Gamma_{2}=\cos (k \phi) r \partial_{t} \\
& \Gamma_{3}=\sin (k \phi) r\left(t \partial_{t}+r \partial_{r}\right) \\
& \Gamma_{4}=\sin (k \phi) r \partial_{t} \\
& \Gamma_{5}=t\left(t \partial_{t}+r \partial_{r}\right) \\
& \Gamma_{6}=\frac{1}{2} r \partial_{r}+t \partial_{t} \\
& \Gamma_{7}=\partial_{t} \\
& \Gamma_{8}=t\left(\cos (k \phi) \partial_{r}-\frac{1}{k r} \sin (k \phi) \partial_{\phi}\right) \\
& \Gamma_{9}=\cos (k \phi) \partial_{r}-\frac{1}{k r} \sin (k \phi) \partial_{\phi}, \\
& \Gamma_{10}=t\left(\sin (k \phi) \partial_{r}+\frac{1}{k r} \cos (k \phi) \partial_{\phi}\right)
\end{aligned}
$$

${ }^{\mathrm{d}} V(r, \phi)=0$.

${ }^{\mathrm{e}} V(r, \phi)=\frac{1}{2} \omega^{2} r^{2}$. 


$$
\begin{aligned}
& \Gamma_{11}=\sin (k \phi) \partial_{r}+\frac{1}{k r} \cos (k \phi) \partial_{\phi}, \\
& \Gamma_{12}=r \partial_{r}, \\
& \Gamma_{13}=k r \cos (2 k \phi) \partial_{r}-\sin (2 k \phi) \partial_{\phi}, \\
& \Gamma_{14}=k r \sin (2 k \phi) \partial_{r}+\cos (2 k \phi) \partial_{\phi}, \\
& \Gamma_{15}=\partial_{\phi} .
\end{aligned}
$$

Consequently system (2.1) is linearizable [6,7], and in order to quantize it we follow the three Steps $1,2,3[8]$ as recalled in the Introduction.

Step 1. We have to find a linearizing transformation which does not alter the time $t$. In [29] it was determined that the linearizing transformation can be found by means of a four-dimensional subalgebra of type $A_{4,5}^{1,1}=\left\langle X_{1}, X_{2}, X_{3}, X_{4}\right\rangle$ in the Mubarakzyanov classification [14,15] with commutation relations:

$$
\left[X_{i}, X_{j}\right]=0, \quad\left[X_{i}, X_{4}\right]=X_{i}, \quad(i, j=1,2,3)
$$

such that $1 \leq \operatorname{rank}\left[X_{1}, X_{2}, X_{3}, X_{4}\right] \leq 3$, and whose canonical form is:

$$
\begin{gathered}
X_{1}=\partial_{\tau}, \quad X_{2}=\partial_{u}, \quad X_{3}=\partial_{v}, \\
X_{4}=\tau \partial_{\tau}+u \partial_{u}+v \partial_{v} .
\end{gathered}
$$

We found that the operators $\Gamma_{7}, \Gamma_{9}, \Gamma_{11}, \Gamma_{16}$, with $\Gamma_{16}=\Gamma_{6}+\frac{1}{2} \Gamma_{12}$, generate such a subalgebra. Consequently the following transformation:

$$
\tau=t, \quad u=r \cos (k \phi), \quad v=r \sin (k \phi) .
$$

takes system (2.1) into the following linear system ${ }^{\mathrm{f}}$ :

$$
\ddot{u}=0, \quad \ddot{v}=0 .
$$

Then the general solution of system (2.1) is:

$$
\begin{aligned}
r & = \pm \sqrt{\left(c_{1} t+c_{2}\right)^{2}+\left(c_{3} t+c_{4}\right)^{2}}, \\
\tan (k \phi) & =\frac{c_{1} t+c_{2}}{c_{3} t+c_{4}},
\end{aligned}
$$

with $c_{i}(i=1,4)$ arbitrary constants.

Step 2. The Lagrangian (1.8) admits eight Noether symmetries, i.e.: $\Gamma_{5}, \Gamma_{6}, \Gamma_{7}, \Gamma_{8}, \Gamma_{9}, \Gamma_{10}, \Gamma_{11}$, $\Gamma_{15}$.

${ }_{\mathrm{f}}$ Namely the Lagrangian equations of a two-dimensional free particle. 
Step 3. The Schrödinger equation of the two-dimensional free particle in the variables $(u, v)$ is

$$
2 \mathrm{i} \psi_{t}+\psi_{u u}+\psi_{v v}=0
$$

with $\psi=\psi(t, u, v)$. If we apply the transformation (2.5), then we obtain that the Schrödinger equation corresponding to system (2.1) is ${ }^{\mathrm{g}}$

$$
2 \mathrm{i} \psi_{t}+\psi_{r r}+\frac{1}{r} \psi_{r}+\frac{1}{k^{2} r^{2}} \psi_{\phi \phi}=0 .
$$

Using the REDUCE programs [16] we find that its Lie point symmetries are generated by the following operators:

$$
\begin{aligned}
& \Lambda_{1}=\Gamma_{15} \\
& \Lambda_{2}=\Gamma_{8}+\mathrm{i} k^{2} r \cos (k \phi) \psi \partial_{\psi}, \\
& \Lambda_{3}=\Gamma_{9}, \\
& \Lambda_{4}=\Gamma_{10}+\mathrm{i} k^{2} r \sin (k \phi) \psi \partial_{\psi}, \\
& \Lambda_{5}=\Gamma_{11}, \\
& \Lambda_{6}=\Gamma_{5}+\frac{1}{2}\left(\mathrm{i} r^{2}-2 t\right) \psi \partial_{\psi}, \\
& \Lambda_{7}=\Gamma_{6}, \\
& \Lambda_{8}=\Gamma_{7}, \\
& \Lambda_{9}=\psi \partial_{\psi}, \quad \Lambda_{\Psi}=\Psi \partial_{\psi},
\end{aligned}
$$

where $\Psi(t, r, \phi)$ is any solution of equation (2.9).

In order to determine the radial Schrödinger equation, we consider the two-dimensional abelian Lie subalgebra generated by the operators

$$
\begin{aligned}
& \Lambda_{\varepsilon}=\Lambda_{8}-\mathrm{i} \varepsilon \Lambda_{9}=\partial_{t}-\mathrm{i} \varepsilon \psi \partial_{\psi} \\
& \Lambda_{\beta}=\Lambda_{1}-\mathrm{i} p \Lambda_{9}=\partial_{\phi}-\mathrm{i} p \psi \partial_{\psi},
\end{aligned}
$$

with $\varepsilon$ and $p$ are arbitrary constants. Then solving the corresponding invariant surface condition [27] yields the following invariant solution

$$
\psi(t, r, \phi)=R(r) e^{-\mathrm{i}(\varepsilon t+p \phi)},
$$

that replaced into (2.9) gives rise to the following radial Schrödinger equation:

$$
R^{\prime \prime}+\frac{1}{r} R^{\prime}+\left(2 \varepsilon-\frac{p^{2}}{k^{2} r^{2}}\right) R=0,
$$

where prime denotes derivative by $r$.

After imposing $p$ to be an integer since the invariant solution (2.12) must be periodic of period $2 \pi$,

$\overline{\mathrm{g}}$ Introducing $\hbar$ into (2.9), i.e.

$$
2 \mathrm{i} \hbar \psi_{t}+\hbar^{2}\left(\psi_{r r}+\frac{1}{r} \psi_{r}+\frac{1}{k^{2} r^{2}} \psi_{\phi \phi}\right)=0
$$

and performing the classical limit [11] yields the Hamilton-Jacobi equation for system (2.1). 
we obtain that the only bounded solution of equation (2.9) is given in terms of the Bessel function of the first kind $J_{\mu}$, i.e.:

$$
\psi_{p, \varepsilon}=J_{\frac{|p|}{k}}(\sqrt{2 \varepsilon}|r|) e^{-\mathrm{i}(\varepsilon t+p \phi)},
$$

with the additional condition $\varepsilon>0$.

\section{Radial harmonic oscillator on the cone}

The Lagrangian equations corresponding to the Lagrangian (1.9) are:

$$
\left\{\begin{array}{l}
\ddot{r}=k^{2} r \dot{\phi}^{2}-\omega^{2} r \\
\ddot{\phi}=-2 \frac{\dot{r} \dot{\phi}}{r} .
\end{array}\right.
$$

Using the REDUCE programs [16] we find that this system admits a fifteen-dimensional Lie point symmetry algebra, isomorphic to $\mathrm{sl}(4, \mathbb{R})$, generated by the following operators:

$$
\begin{aligned}
& \Xi_{1}=\cos (k \phi) r\left(\cos (\omega t) \partial_{t}-\omega r \sin (\omega t) \partial_{r}\right), \\
& \Xi_{2}=\cos (k \phi) r\left(\sin (\omega t) \partial_{t}+\omega r \cos (\omega t) \partial_{r}\right), \\
& \Xi_{3}=\sin (k \phi) r\left(\cos (\omega t) \partial_{t}-\omega r \sin (\omega t) \partial_{r}\right), \\
& \Xi_{4}=\sin (k \phi) r\left(\sin (\omega t) \partial_{t}+\omega r \cos (\omega t) \partial_{r}\right), \\
& \Xi_{5}=\cos (2 k \phi) r \partial_{r}-\frac{1}{k} \sin (2 k \phi) \partial_{\phi}, \\
& \Xi_{6}=\sin (2 k \phi) r \partial_{r}+\frac{1}{k} \cos (2 k \phi) \partial_{\phi}, \\
& \Xi_{7}=r \partial_{r}, \\
& \Xi_{8}=\partial_{\phi}, \\
& \Xi_{9}=\partial_{t}, \\
& \Xi_{10}=\cos (2 \omega t) \partial_{t}-\omega \sin (2 \omega t) r \partial_{r}, \\
& \Xi_{11}=\sin (2 \omega t) \partial_{t}+\omega \cos (2 \omega t) r \partial_{r}, \\
& \Xi_{12}=\cos (\omega t)\left(\cos (k \phi) \partial_{r}-\frac{1}{k r} \sin (k \phi) \partial_{\phi}\right), \\
& \Xi_{13}=\sin (\omega t)\left(\cos (k \phi) \partial_{r}-\frac{1}{k r} \sin (k \phi) \partial_{\phi}\right), \\
& \Xi_{14}=\cos (\omega t)\left(\sin (k \phi) \partial_{r}+\frac{1}{k r} \cos (k \phi) \partial_{\phi}\right), \\
& \Xi_{15}=\sin (\omega t)\left(\sin (k \phi) \partial_{r}+\frac{1}{k r} \cos (k \phi) \partial_{\phi}\right) .
\end{aligned}
$$

Consequently system (3.1) is linearizable [6,7], and in order to quantize it we follow the three Steps $1,2,3[8]$ as recalled in the Introduction. 
Step 1. The transformation (2.5) applied to (3.1) yields:

$$
\ddot{u}+\omega^{2} u=0, \quad \ddot{v}+\omega^{2} v=0,
$$

namely the equations of a two-dimensional linear harmonic oscillator. Therefore, the general solution of (3.1) is:

$$
\begin{aligned}
r & = \pm \sqrt{\left(c_{1} \cos (\omega t)+c_{2} \sin (\omega t)\right)^{2}+\left(c_{3} \cos (\omega t)+c_{4} \sin (\omega t)\right)^{2}} \\
\tan (k \phi) & =\frac{c_{1} \cos (\omega t)+c_{2} \sin (\omega t)}{c_{3} \cos (\omega t)+c_{4} \sin (\omega t)} .
\end{aligned}
$$

Step 2. The Lagrangian (1.9) admits eight Noether symmetries, namely $\Xi_{i}$ with $i=8, \ldots, 15$ in (3.2).

Step 3. The Schrödinger equation for a two-dimensional linear harmonic oscillator in the variables $(u, v)$ and wave function $\psi$ is:

$$
2 \mathrm{i} \psi_{t}+\psi_{u u}+\psi_{v v}-\omega^{2}\left(u^{2}+v^{2}\right) \psi=0
$$

If we apply the transformation (2.5), then the Schrödinger equation corresponding to system (3.1) is $^{\text {h: }}$

$$
2 \mathrm{i} \psi_{t}+\psi_{r r}+\frac{1}{r} \psi_{r}+\frac{1}{k^{2} r^{2}} \psi_{\phi \phi}-\omega^{2} r^{2} \psi=0 .
$$

Using the REDUCE programs [16] we find that its Lie point symmetries are generated by the following operators:

$$
\begin{aligned}
& \Omega_{1}=\Xi_{8}, \\
& \Omega_{2}=\Xi_{10}+\omega\left(\sin (2 \omega t)-2 \mathrm{i} \cos (2 \omega t) \omega r^{2}\right) \psi \partial_{\psi}, \\
& \Omega_{3}=\Xi_{11}-\omega\left(\cos (2 \omega t)+2 \mathrm{i} \sin (2 \omega t) \omega r^{2}\right) \psi \partial_{\psi}, \\
& \Omega_{4}=\Xi_{9}, \\
& \Omega_{5}=\Xi_{14}-\mathrm{i} \omega r \sin (\omega t) \sin (k \phi) \psi \partial_{\psi}, \\
& \Omega_{6}=\Xi_{15}+\mathrm{i} \omega r \cos (\omega t) \cos (k \phi) \psi \partial_{\psi}, \\
& \Omega_{7}=\Xi_{12}-\mathrm{i} \omega r \sin (\omega t) \cos (k \phi) \psi \partial_{\psi}, \\
& \Omega_{8}=\Xi_{13}+\mathrm{i} \omega r \cos (\omega t) \cos (k \phi) \psi \partial_{\psi}, \\
& \Omega_{9}=\psi \partial_{\psi}, \quad \Omega \Psi=\Psi \partial_{\psi},
\end{aligned}
$$

where $\Psi=\Psi(t, r, \phi)$ is any solution of (3.6).

Equation (3.6) is the same Schrödinger equation that was obtained in $[1,5,13]$ in the case that $r \in$ $(0, \infty)$. Therefore the eigenfunctions of equation (3.6) are given in terms of the associated Laguerre

$\overline{\mathrm{h}}$ Introducing $\hbar$ into (3.6), i.e.

$$
2 \mathrm{i} \hbar \psi_{t}+\hbar^{2}\left(\psi_{r r}+\frac{1}{r} \psi_{r}+\frac{1}{k^{2} r^{2}} \psi_{\phi \phi}\right)-\omega^{2} r^{2} \psi=0,
$$

and performing the classical limit [11] yields the Hamilton-Jacobi equation for system (3.1). 
polynomials $L_{n}^{\mu}$, i.e.:

$$
\psi_{n, p}=e^{-\mathrm{i} E_{n} t+\mathrm{i} p \phi}|r|^{|p| / k} e^{-(1 / 2) \omega r^{2}} L_{n}^{|p| / k}\left(\omega r^{2}\right), \quad(n \in \mathbb{N}, p \in \mathbb{Z})
$$

with eigenvalues

$$
E_{n}=\omega\left(2 n+\frac{|p|}{k}+1\right)
$$

\section{Comparison of the different outcomes}

The quantization of the harmonic oscillator on a cone with one nappe has been studied in several papers, e.g. $[1,5,13]$.

Our Schrödinger equation (3.6) is exactly the same that all the above authors derived by means of the DeWitt's approach [4], namely Noether symmetries of the classical Lagrangian systems are preserved as we shown.

In [10], the authors derived a different Schrödinger equation for both the free particle and the harmonic oscillator on the double cone. They took the usual angular momentum operator, i.e., $\hat{p}_{\phi}=$ $-\mathrm{i} \partial_{\phi}$, and looked for a self-adjoint operator for the radial momentum of the type

$$
\hat{p}_{r}=-\mathrm{i}\left(\partial_{r}+F(r)\right),
$$

with respect to the scalar product

$$
\langle f, g\rangle=\int_{0}^{2 \pi} \int_{-\infty}^{\infty} f^{*}(r, \phi) g(r, \phi)|r| \mathrm{d} r \mathrm{~d} \phi,
$$

on the space of square integrable functions $f(r, \phi), g(r, \phi)$ on the cone. This yields that the selfadjoint operator is:

$$
\hat{p}_{r}=-\mathrm{i}\left(\partial_{r}+\frac{1}{2 r}\right),
$$

and consequently, without any further assumption, the Schrödinger equation for the free particle was derived to be:

$$
2 \mathrm{i} \psi_{t}+\psi_{r r}+\frac{1}{r} \psi_{r}+\frac{1}{k^{2} r^{2}} \psi_{\phi \phi}-\frac{1}{4 r^{2}} \psi=0,
$$

while the Schrödinger equation for the harmonic oscillator became:

$$
2 \mathrm{i} \psi_{t}+\psi_{r r}+\frac{1}{r} \psi_{r}+\frac{1}{k^{2} r^{2}} \psi_{\phi \phi}-\left(\frac{1}{4 r^{2}}+\omega^{2} r^{2}\right) \psi=0 .
$$

Both equations (4.4) and (4.5) do not preserve the Noether symmetries of the free particle and the harmonic oscillator on the cone, respectively. Indeed the Lie symmetries of equation (4.4) are:

$$
\begin{aligned}
& \Upsilon_{1}=\Gamma_{15}, \\
& \Upsilon_{2}=\Gamma_{5}+\frac{1}{2}\left(i r^{2}-2 t\right) \psi \partial_{\psi}, \\
& \Upsilon_{3}=\Gamma_{6}, \\
& \Upsilon_{4}=\Gamma_{7},
\end{aligned}
$$




$$
\Upsilon_{5}=\psi \partial_{\psi}, \quad \Upsilon_{\Psi}=\Psi \partial_{\psi},
$$

with $\Psi=\Psi(t, r, \phi)$ any solution of (4.4), while those of equation (4.5) are:

$$
\begin{aligned}
& \Pi_{1}=\Xi_{8}, \\
& \Pi_{2}=\Xi_{11}-\omega\left(\cos (2 \omega t)+2 \mathrm{i} \sin (2 \omega t) \omega r^{2}\right) \psi \partial_{\psi}, \\
& \Pi_{3}=\Xi_{10}+\omega\left(\sin (2 \omega t)-2 \mathrm{i} \cos (2 \omega t) \omega r^{2}\right) \psi \partial_{\psi}, \\
& \Pi_{4}=\Xi_{9}, \\
& \Pi_{5}=\psi \partial_{\psi}, \quad \Pi_{\Psi}=\Psi \partial_{\psi},
\end{aligned}
$$

with $\Psi=\Psi(t, r, \phi)$ any solution of (4.5). Indeed, the additional term $-\psi / 4 r^{2}$ in both equations (4.4) and (4.5) breaks the symmetries, i.e. four out of eight symmetries are not preserved. Therefore, our eigenfunctions are different from those obtained in [10] although the boundary conditions are the same. Moreover, in the case of the harmonic oscillator the eigenvalues that were derived in [10] are:

$$
E_{n}=\omega\left(2 n+\frac{1}{2} \sqrt{1+\frac{4 p^{2}}{k^{2}}}+1\right),
$$

instead of those that we derived, i.e. (3.9):

$$
E_{n}=\omega\left(2 n+\frac{|p|}{k}+1\right)
$$

We would like to underline that the eigenvalues (3.9) that we have obtained coincide with those derived by other authors, e.g. $[1,5,13]$, if one nappe only is considered. As observed by an anonymous referee, one cannot distinguish locally between a double cone and that with a single nappe. Consequently, the quantization method that preserves the Noether symmetries of the classical problem is consistent with this observation.

Moreover, if we introduce the self-adjoint momentum operators as defined by DeWitt in [4], i.e.:

$$
\hat{p}_{k}=-\mathrm{i}\left(\frac{\partial}{\partial_{q_{k}}}+\Gamma_{k j}^{j}\right)
$$

with $\Gamma_{j k}^{j}$ the contracted Christoffel symbols, then we obtain the same angular and radial momenta in [10]. The different outcome is in the Hamiltonian operator that shall include an extra "quantum mechanical potential" $Q$ which must be added to the covariant classical Hamiltonian in order to produce the covariant quantum Hamiltonian [4]. Adding this potential $Q$ means to eliminate the symmetry-breaking term $-\psi / 4 r^{2}$ in (4.4) and (4.5), and consequently the Schrödinger equations (2.9) and (3.6) are obtained. This shows that the quantization method that preserves the Noether symmetries of the classical problem corresponds to DeWitt's approach.

\section{Conclusions}

In this paper we have derived the Schrödinger equation for both the free particle and the harmonic oscillator on a double cone by requiring the preservation of the Noether symmetries of the classical problem. Indeed the Noether symmetries admitted by the Lagrangian (1.8) are the Lie symmetries 
of the Schrödinger equation (2.9), and the Noether symmetries admitted by the Lagrangian (1.9) are the Lie symmetries of the Schrödinger equation (3.6). In particular the latter coincides with the Schrödinger equation derived by other authors, e.g. [1,5,13], in the case of a single cone. On the other hand, the Schrödinger equations for the same problems that were obtained in [10] do not preserve the Noether symmetries of the classical problem and therefore yield results quite different from the ones we have derived here.

Further insight is needed especially from the experimentalists as recently stated in [12]. However, in 1955 Schrödinger wrote [28]:

The accepted foundation of q.m. claims to be intimately linked with experimental science. But actually it is based on a scheme of measurement which, because it is entirely antiquated, is hardly fit to describe any relevant experiment that is actually carried out, but a host of such as are for ever confined to the imagination of their inventors.

\section{Acknowledgements}

GG is supported by INFN IS-CSN4 Mathematical Methods of Nonlinear Physics.

MCN acknowledges the support of the Italian Ministry of University and Scientific Research through PRIN 2010-2011, Prot. 2010JJ4KPA_004, Geometric and analytic theory of Hamiltonian systems in finite and infinite dimensions.

\section{References}

[1] M.H. Al-Hashimi, W.J. Wiese, Runge-Lenz vector, accidental $S U(2)$ symmetry, and unusual multiplets for motion on a cone, Ann. Phys. 323 (2008) 82-104.

[2] M. Błaszak, Z. Domański, Canonical transformations in quantum mechanics, Ann. Phys. 331 (2013) 70-96.

[3] Y. Brihaye, P. Kosiński, P. Maślanka, Dynamics on the cone: Closed orbits and superintegrability, Ann. Phys. 344 (2014) 253-262.

[4] B.S. DeWitt, Point transformations in Quantum Mechanics, Phys. Rev. 85 (1952) 653-661.

[5] C. Furtado, F. Moraes, Harmonic oscillator interacting with conical singularities, J. Phys. A: Math. Gen. 33 (2000) 5513-5519.

[6] F. González-Gascón, A. González-López, Symmetries of differential equations, J. Math. Phys. 24 (1983) 2006-2021.

[7] A. González-López, Symmetries of linear systems of second-order ordinary differential equations, J. Math. Phys. 29 (1988) 1097-1105.

[8] G. Gubbiotti, M.C. Nucci, Noether symmetries and the quantization of a Liénard-type nonlinear oscillator, J. Nonlinear Math. Phys. 21 (2014) 248-264.

[9] G. Gubbiotti, M.C. Nucci, Quantization of quadratic Liénard-type equations by preserving Noether symmetries, J. Math. Anal. Appl. 422 (2015) 1235-1246.

[10] K. Kowalski, J. Rembielński, On the dynamics of a particle on a cone, Annals of Physics 329 (2013) 146-157.

[11] L.D. Landau, E.M. Lifshitz, Quantum mechanics. Non-relativistic theory Vol.3. Pergamon Press, Oxford, 1991.

[12] M.D. Lopes, S. Azevedo, F. Moraes, M. Machado, Theoretical study of carbon double cones, Eur. Phys. J. B 88 (2015) 10.

[13] A.J. Makowski, Quantum-classical correspondence for motion on a plane with deficit angle, Ann. Phys. 325 (2010) 1622-1632.

[14] G. Mubarakzyanov, On solvable Lie algebras, Izv. Vys. Ucheb. Zaved. Matematika 32 (1963) 114-123.

[15] G. Mubarakzyanov, Certain theorems on solvable Lie algebras, Izv. Vys. Ucheb. Zaved. Matematika 55 (1966) 95-98. 
[16] M.C. Nucci, Interactive REDUCE programs for calculating Lie point, non-classical, Lie-Bäcklund, and approximate symmetries of differential equations: manual and floppy disk, in: N. Ibragimov (Ed.), CRC Handbook of Lie Group Analysis of Differential Equations, Vol. III: New Trends in Theoretical Developments and Computational Methods, CRC Press, Boca Raton, 1996, 415-481.

[17] M.C. Nucci, Quantization of classical mechanics: shall we Lie?, Theor. Math. Phys. 168 (2011) 9941001.

[18] M.C. Nucci, From Lagrangian to Quantum Mechanics with Symmetries, J. Phys.: Conf. Ser. 380 (2012) 012008.

[19] M.C. Nucci, Quantizing preserving Noether symmetries, J. Nonlinear Math. Phys. 20 (2013) 451-463.

[20] M.C. Nucci, Symmetries for thought, Math. Notes Miskolc 14 (2013) 461-474.

[21] M.C. Nucci, Spectral realization of the Riemann zeros by quantizing $H=w(x)\left(p+\ell_{p}^{2} / p\right)$ : the LieNoether symmetry approach, J. Phys.: Conf. Ser. 482 (2014) 012032.

[22] M.C. Nucci, Ubiquitous symmetries, Theor. Math. Phys. 188 (2016) 1361-1370.

[23] M.C. Nucci, P.G.L. Leach, Lagrangians galore, J. Math. Phys. 48 (2007) 123510 .

[24] M.C. Nucci, P.G.L. Leach, Jacobi last multiplier and Lagrangians for multidimensional linear systems, J. Math. Phys. 49 (2008) 073517.

[25] M.C. Nucci, K.M. Tamizhmani, Using an old method of Jacobi to derive Lagrangians: a nonlinear dynamical system with variable coefficients, Nuovo Cimento B 125 (2010) 255-269.

[26] M.C. Nucci, K.M. Tamizhmani, Lagrangians for dissipative nonlinear oscillators: the method of Jacobi Last Multiplier, J. Nonlinear Math. Phys. 17 (2010) 167-178.

[27] P.J. Olver, Applications of Lie Groups to Differential Equations, Springer-Verlag, Berlin, 1986.

[28] E. Schrödinger, The Philosophy of Experiment, Il Nuovo Cimento 1 (1955) 5-15.

[29] C. Soh, F. Mahomed, Linearization criteria for a system of second-order ordinary differential equations, Int. J. Non-lin. Mech. 36 (2001) 671-677.

[30] L. van Hove, Sur certaines représentations unitaires d'un groupe infini de transformations, Memoires de la Académie Royale Belgique, Classe de Sciences 26 (1951) 1-102. 